\title{
General introduction to microstructural evolution under cascade damage conditions*
}

\author{
Hartmut Wiedersich \\ Materials Science Division \\ Argonne National Laboratory \\ Argonne, IL 60439
}

June 1993

\begin{tabular}{|c|}
\hline 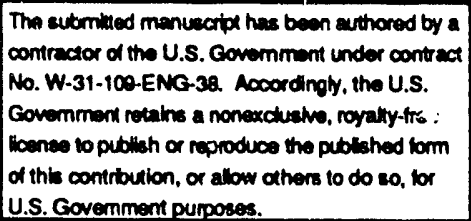 \\
\hline
\end{tabular}

AECEIVISO

JUL 261993

OSTI

\section{DISCLAIMER}

\begin{abstract}
This report was prepared as an account of work sponsored by an agency of the United States Government. Neither the United States Government nor any agency thereof, nor any of their employees, makes any warranty, express or implied, or assumes any legal liability or responsibility for the accuracy, completeness, or usefulness of any information, apparatus, product, or process disclosed, or represents that its use would not infringe privately owned rights. Reference herein to any specific commercial product, process, or service by trade name, trademark, manufacturer, or otherwise does not necessarily constitute or imply its endorsement, recommendation, or favoring by the United States Government or any agency thereof. The views and opinions of authors expressed herein do not necessarily state or reflect those of the United States Government or any agency thereof.
\end{abstract}

To be published in the Proceedings of the International Workshop on Time Dependence of Radiation Damage Accumulation and its Impact on Materials Properties, October 14-20, 1992, Montreux, Switzerland.

*Work supported by the U. S. Department of Energy, BES-Materials Sciences, under Contract W-31-109-Eng-38. 
General introduction to microstructural evolution under cascade damage conditions*

\author{
Hartmut Wiedersich \\ Materials Science Division \\ Argonne National Laboratory \\ Argonne, IL 60439
}

\title{
Abstract
}

A short overview of the processes that affect the evolution of the microstructure during irradiation is given. The processes include defect production with an emphasis on the effects of the dynamic cascade events, defect clustering, irradiation-enhanced diffusion, radiation-induced segregation, phase decompositions and phase transformations. A simple model for the description of the development of the defect microstructure in a pure metal during cascade producing irradiation is also outlined which can provide, in principle, defect fluxes required for the description of other microstructural processes such as phase decomposition and irradiation-induced precipitation.

*Work supported by the U. S. Department of Energy, BES-Materials Sciences under Contract W-31-109-Eng-38 


\section{Introduction}

The evolution of the microstructure during irradiation with energetic neutrons, ions or electrons is complex because of the intricate interaction of a number of processes. For detailed descriptions of various aspects of irradiation microstructures and contributing mechanisms, the reader is referred to $\bar{a}$ number of recent conference proceedings and a volume in the series "Modern Problems in Condensed Matter Sciences" [1-5]. Here we will give only a short overview of the processes involved.

Primary knock-on atoms (PKAs) are generated by interactions of energetic incoming particles with lattice atoms. At low transferred energy, isolated interstitial atom-vacancy pairs result. If of sufficient energy, PKAs produce generations of secondary knock-on atoms which contribute to the generation of defects in form of displacement cascades. Significant mutual annihilation of opposite types of defects as well as clustering of like defects occurs within the lifetime of cascades (i. e., within a few ps).

Interstitials, vacancies and small clusters of these defects are thermally mobile to various degrees except at rather low temperatures. Individual defects are eliminated via a number of reactions, e. g., by annihilation at dislocations, interfaces and surfaces, by mutual recombination, or by incorporation into defect clusters. These processes lead to the development of a temperature, dose and dose rate dependent defect and dislocation microstructure that may contain voids.

Displacement events lead to local mixing and disordering in alloys and the corresponding changes in chemical potential of the elements. Also the forced presence of excess point defects changes the free energy of phases. As a consequence, phase decomposition, precipitate dissolution, or phase transformations 
to thermally metastable phases may occur.

The presence of mobile point defects in excess of their thermal equilibrium concentrations leads to an enhancement of diffusion processes. Thus, equilibration processes such as phase decomposition or precipitate coarsening, which may be frozen at the ambient temperature, can be vastly accelerated by irradiation. However, the dynamic displacement processes (with and without cascades) locally rearrange atoms in a random fashion which often counteracts thermal equilibration.

Defect production during irradiation with energetic neutrons occurs uniformly and randomly throughout the material on appropriate length and time scales. For example, atoms in the cladding or structural material in a reactor core will be displaced, on average, with equal frequency throughout the microstructure despite the occurrence of cascades. However, defect annihilation at stationary sinks ( such as grain boundaries, network dislocations and voids ) is spatially nonrandom. Therefore, persistent defect currents flow to these sinks. Preferential coupling of alloying elements to the defect fluxes enriches or depletes such elements near the defect sinks, i.e., causes radiation-induced segregation. Local changes in alloy composition may induce phase separation or, conversely, dissolution of secondary phases.

The simultaneous action of a number of the processes outlined above makes the evolution of microstructures during irradiation exceedingly complex. Therefore, no comprehensive, quantitative description has been attempted as yet although the individual contributing processes are reasonably well understood. Of particular importance is the development of means by which to predict microstructural developments in alloys under irradiations characteristic of future fusion reactors from our knowledge derived from irradiations in presently accessible irradiation 
environments.

In the following, we will summarize the characteristics of the individual processes contributing to microstructural development in the light of the present knowledge of microstructural evolution. First defect production and cascade characteristics will be discussed. In section 3 the main features of microstructures consisting of defect clusters, dislocations and voids will be outlined. Phase changes and their relations to alloying element redistribution will be summarized in section 4 , followed by an outline of a simple model for the evolution of defect microstructures in section 5 .

\section{Displacen.ent Cascade Characteristics}

Primary knock-on energies from the threshold displacement energy up to about $1 \mathrm{keV}$ produce isolated defect pairs or small groups of defects. As the PKA energy increases, an increasing number of nearby atoms receive sufficient transferred energy to generate further generations of knock-on atoms, and a correspondingly larger number of displaced atoms is generated. The cascade formation mechanism has been studied by computer simulations using the binary collision approximation (see, e.g., M. Robinson and I. M. Torrens, Heinisch et al., [6-8]) and in more detail by molecular dynamics calculations (see, e.g., Diaz de la Rubia et al., English et al., [9-11]).

The sequence of events can be characterized as follows [9]: The energy of the primary knock-on atom is distributed over a large number of atoms in close proximity in a few tenths of a picosecond. A number of atoms is transported directly or via replacement sequences to the periphery of the cascade region forming interstitial atoms. The atomic positions in the central region become uncorrelated 
to the original lattice positions and the energy density exceeds greatly that of a molten region. Within about a picosecond equipartition between kinetic and potential energy is approached; thus, this region may be best described as a highly superheated and compressed melt. As the localized energy is dissipated into the surrounding matrix the 'melt' crystallizes from the periphery to the center. The vacancies resulting from the ejected atoms condense into one or more clusters in the central region. Concurrently much atomic rearrangement proceeds throughout the entire cascade region, leading to recombination and to clustering of defects, particularly, of interstitial atoms in the peripheral region. After several picoseconds the cascade region has returned to ambient temperature.

Experimental observations by transmission electron microscopy (TEM) have confirmed much of the remnant structure of a cascade (see, e.g., Kirk et al. [13], Kiritani, [14-15]). In particular, collapsed faulted and unfaulted dislocation loops, and stackingfault tetrahedra of vacancy type are frequently observed as remainders of cascades. TEM evidence for the simultaneous presence of small interstitial defect, clusters is not as direct, as discussed by Wilkins [16]. However, Rauch et al. and Peisl et al. have shown, by diffuse $x$-ray scattering, that interstitial and vacancy clusters are present after low temperature neutron irradiations [17-18].

As the primary recoil energy increases above about 15 to $20 \mathrm{keV}$, the cascades tend to break up into closely spaced subcascades (Merkle [19], Kiritani [14], De la Rubia and Guinan [10]). With increasing energy, more subcascades are formed within a group with an average energy of about $15 \mathrm{keV}$ [14]. The characteristics of the subcascades presumably resemble those of individual cascades described above.

If the ambient temperature is sufficient for thermal mobility, point defects and small defect clusters will be released from cascade regions into the surrounding 
matrix. Defect clusters f frmed in cascades will subsequently grow and/or shrink by absorption of mobile defects and by thermal emission of point defects. Because of the randomness of these events, sizes of individual clusters will undergo significant fluctuations over time.

Whereas independent nucleation of vacancy and of interstitial clusters does not seem necessary in materials under cascade producing irradiations, nucleation of such clusters from excess point defects in the matrix with and without insoluble gas should not be ruled out. For example, the fact that interstitial dislocation loops and voids are produced in many materials under $\mathrm{MeV}$ electron irradiations at elevated temperatures shows that these defect clusters nucleate in matrices supersaturated solely with point defects (see, e.g., Urban et al. [20]).

\section{Evolution of the defect microstructure}

Continued irradiation at low temperatures produces highly dense defect structures consisting of both interstitial and vacancy type defect clusters. These types of structures develop for electron and cascade producing irradiations even at temperatures at which interstitials are highly, and vacancies are moderately mobile (stage III annealing). [16] Little detailed knowledge of these structures, sometimes called black death in electron microscopy, seems to be available.

At higher temperatures, above about 0.3 of the absolute melting temperature $\mathrm{T}_{\mathrm{m}}$, the coarser components of the microstructures are easily identified by electron microscopy. In annealed materials, dislocation loops, predominately interstitial in nature, form and grow; loops interact with each other and preexisting network dislocations and form a dislocation network. The density of the network is characteristic of the temperature of the irradiation. Small voids appear and grow. 
Their number density tends to saturate at values also characteristic of the temperature. In cold-worked materials with a high initial dislocation density, the dislocation networks rearrange during irradiation and approach a steady state density characteristic of the temperature. Again small voids appear and grow, usually after a somewhat longer incubation dose than that observed in annealed materials.

The defect microstructures tend to become typical of the temperature and type of irradiation at high doses independent of the initial state (see, e.g., the papers by Garner [21], Zinkle [22] and Little [23] in this Proceedings). The defect structures are very dense below and near the lower end of the void swelling regime and undergo a transition to low density around the peak swelling temperature.

Although in most cases the defect microstructure appears to be random, spatially regular defect arrangements have been frequently observed: Void lattices that assume the symmetry and orientation of the host lattice; ordered stackingfault tetrahedra and periodic dislocation walls (see, e.g., J. H. Evans [24] and Jäger and Trinkaus [25]).

\section{Phase transformations}

Phase transformations can be induced by irradiation. Perhaps the simplest types are order-disorder transformations. At low temperature with no or limited defect mobilities, displacements and replacement sequences increase the disorder in initially ordered phases. With increasing temperature and increased defect mobilities, thermal migration of defects counteracts the disordering effects of the displacement processes. Eventually a quasi-steady-state of order should prevail. A good example of these effects is given in the work by Urban and coworkers on 
Ni 4 Mo which estabiished a phase diagram of this intermetallic alloy under electron irradiation. In fact, two different types of order occur during irradiation, indicating that irradiation can stabilize metastable phases kinetically. [26], [27]

Another set of radiation-induced phase transformations are crystalline-toamorphous phase transitions. Again the displacement processes tend to disorder the crystalline arrangement of atoms. The dose required for amorphization has only a small temperature dependence below a limiting "amorphization temperature, $\mathrm{T}_{\mathrm{a}}$ " above which amorphization cannot be induced. Increased defect mobility at higher temperature counteracts the displacement disordering. The temperature $T_{\mathbf{a}}$ is higher for cascade producing irradiation than for electron irradiation, indicating that higher atomic mobilities are required to recrystallize the highly disordered regions created in the center of cascades than to eliminate defects produced isolated in low energy events. $[28,29]$

Up to now we touched only on phase transformations that occur without local compositional changes. Two classes of radiation induced phase changes involving compositional changes on a microstructural level have been recognized. The first can be characterized as "chemically driven". The presence of radiation produced defects increases the free energy of a phase and, therefore, the phase field of thermodynamically stable phases may be shifted, and a single phase alloy may decompose into two phases. The radiation-induced precipitation of $\mathrm{Zn}$ in Aluminum investigated experimentally and theoretically by Martin and coworkers is an example [30]. The reverse, e. g., the radiation-induced dissolution of intermetallic precipitates on account of the change in order under irradiation seems also likely, since the increase in chemical potential of the solute in the disordered precipitate should increase its solubility in the matrix. [31] 
The second class of phase transformation with compositional changes can be characterized as "mass transport" or "radiation-induced segregation" driven transformations. [32] In these cases the preferential coupling of alloying elements to persistent defect fluxes modifies the composition of the alloy in the neighborhood of defect sinks (and sources if defect production is spatially not uniform as, e. g., during ion bombardment). Phase decomposition or precipitate dissolution is predominately a consequence of the change in local composition; local deviations of defect concentrations from thermal equilibrium are not likely to contribute significantly to the chemical forces involved.

\section{Model description of microstructural evolution during irradiation}

It is apparent that a complete description of microstructural development under irradiation will need a rather complex model because of the large variety of processes involved, whose effects often oppose each other. One important aim of such a model is to be able to predict and compare the microstructural characteristics resulting from irradiations with different primary knock-on spectra. Comparison on the basis of the total number of displaced atoms calculated, e. g., by the NRT method [33] is grossly inadequate.

One approach to improve correlations between microstructures has been to introduce the concept of long-range migrating or free defects. It has been well established that processes, such as radiation-induced segregation and self-diffusion, that are mitigated by the motion of point defects, are significantly less enhanced by irradiations with heavy ions (or energetic neutrons) than by irradiations with electrons or light ions. For example, the efficiency of inducing segregation in $\mathrm{Ni}(\mathrm{Si})$ alloys decreases dramatically from that of protons via that of $\mathrm{He}$ and $\mathrm{Li}$ ions to that 
of $\mathrm{Ni}$ and $\mathrm{Kr}$ ions [34-36]. The efficiency of the last two ions is at most a few percent of that observed for protons when compared at the same calculated dose in displacements per atom (dpa). Similarly, measurements of self-diffusion in Ni and diffusion of $\mathrm{Ni}$ in $\mathrm{Cu}$ show that only about $1.5 \%$ of the calculated displaced atoms contribute to radiation-enhanced diffusion during $300 \mathrm{keV}$ self-ion irradiation $[37,38]$.

A quantitative treatment of long-range migrating defects (often called free defects) is an important step in devising a comprehensive model for the microstructural evolution since many of the processes involve migration of radiation-produced defects. If one assumes that during irradiation at elevated temperatures additional intracascade recombination reduces the population of freely migrating defects to the values deduced from radiation-induced segregation or radiation-enhanced diffusion studies, one finds that almost no defects could escape from cascades producing more than about 5 defect pairs [39]. Along similar lines, Naundorf et al. $[40,41]$ showed that single defect pairs created at a sufficient distance (larger than the spontaneous recombination radius) from any other defects in a cascade would account for the observed magnitude of freely migrating defect fraction. Neither of these approaches takes into account that a significant fraction of the defects surviving the cascade event are in the form of small defect clusters which not only remove defects from those available for long-range migration, but also contribute to the sink strength and, therefore, reduce the lifetime of defects released into the matrix $[42,43]$. It should be emphasized that the quantity important for microstructural evolution is the "free defect population", i.e., the product of the number of defects released into the matrix and the average number of jumps the defects undergo before annihilation, rather than the number of defects 
released per se.

In the following we will outline a m.odel that describes the evolution of defect clusters formed in cascades in a simple material. It shows that the defert clusters contribute significantly to the elimination of mobile defects and are, in fact, the predominate sinks at low and intermediate temperatures. Hence, defect clusters need to be taken into account when estimating the freely migrating defect population. A more detailed description and results of model calculations can be found in reference [44].

Defects are produced in cascades. A cascade is characterized by the total number, $\mathrm{N}_{\mathbf{0}}$, of defects produced by collisional processes (NRT value, [33]). A fraction of defects, $f_{r}$, recombines (equal for vacancies, $v$, and interstitials, $i$ ); fractions $f_{v c l}$ and $f_{i c l}$ of vacancies and interstitials, respectively, form immobile clusters; the remaining vacancies and interstitials, fractions $f_{v}$ and $f_{i}$, enter the matrix as freely migrating defects. Note, only three of the five fractions $f_{v}, f_{i}, f_{r}, f_{v c l}$ and ficl are independent. The clusters formed provide sinks in addition to the conventional matrix sinks - dislocations, grain boundaries, voids and surfaces. Clusters grow by acquisition of freely migrating defects of the same type, and shrink by the absorption of the opposite type of defects. During formation of a cascade, the central region corresponding to the 'molten' zone obliterates any pre-existing defect clusters (and free vacancies and interstitials) contained in the zone. The net number of obliterated defects, together with the fraction $f_{v c l}$ of newly produced vacancies, reappears on resolidification of the zone as a single defect cluster. The new interstitial clusters created by the cascade (containing the fraction ficl of $\mathrm{N}_{0}$ ) are assumed to be formed outside of the molten zone. 
With this set of assumptions, rate equations for the free defect concentrations can be set up and solved for steady state. Using the defect fluxes obtained, and the production and dissolution rates of clusters by cascades, the evolution of cluster size distributions can be integrated step-wise, recalculating the changing sink strength and the free defect fluxes in the steady-state approximation after each time step.

The rate equations for the time rate of change in the concentrations of point defects are somewhat modified from the usual form $[45,46]$ :

$$
\mathrm{dc}_{i, \mathrm{v}} / \mathrm{dt}=\mathrm{k}_{\mathrm{i}, \mathrm{v}}-\operatorname{Ac}_{\mathrm{i}} \mathrm{q}_{\mathrm{v}}-\left(v_{i, \mathrm{v}} p_{i, \mathrm{v}}+\mathrm{k}_{\mathrm{m}}\right) c_{i, \mathrm{v}}
$$

where $c_{i, v}$ is the concentration, in atomic fraction, of freely migrating interstitials or vacancies $(i, v)$, respectively; $k_{i, v}$ is the production rate of freely migrating interstitials or vacancies from all sources (cascades, thermal emission from sinks including clusters, and from clusters that shrink below their minimum stable, immobile size); $\mathrm{A}$ is the recombination rate constant containing the spontaneous recombination radius and the jump frequencies $v_{i, v}$ of defects; pi,v is the sink annihilation probability per defect jump at all sinks including immobile defect clusters; and $\mathbf{k}_{\mathbf{m}}$ is the rate at which lattice sites are subjected to 'melting' in the central regions of cascades.

A few comments need to be made to emphasize the diffe. ences of eqs. (1) from the usual rate equations: the production rate of freely migrating interstitials is different from that of freely migrating vacancies because (i) of the different numbers of interstitials and vacancies lost to clusters in cascades and because (ii) of the greater excess rate of evaporation of vacancies fror sinks, especially from small vacancy clusters. In fact, $k_{v}$ can be greater or smaller than $k_{i}$ depending on the 
prevailing cluster populations and temperature. For a given cluster population, $\mathbf{k}_{\mathbf{v}}$ $k_{i}$ may change sign as a function of temperature. The term involving elimination of freely migrating defects in the 'molten' zone, $\mathrm{k}_{\mathrm{m}} \mathrm{ci,v}$, limits the population of the slower moving defect species at low temperatures.

The rate of change in the concentration of immobile defect clusters of a given size, $n$, and species, e.g., vacancy clusters, can be expressed by

$$
\begin{gathered}
\mathrm{dm}_{\mathrm{v}, \mathrm{n}} / \mathrm{dt}=\mathrm{k}_{\text {cas, }, n}+\left(j_{i, n+1}+e_{\mathrm{v}, \mathrm{n}+1}\right) \mathrm{m}_{\mathrm{v}, \mathrm{n}+1}+j_{\mathrm{v}, \mathrm{n}-1} \mathrm{~m}_{\mathrm{v}, \mathrm{n}-1} \\
-\left(j_{i, n}+j_{\mathrm{v}, \mathrm{n}}+\mathrm{e}_{\mathrm{v}, \mathrm{n}}+\mathrm{d}_{\mathrm{cas}, \mathrm{vn}}\right) \mathrm{m}_{\mathrm{v}, \mathrm{n}}
\end{gathered}
$$

Here, $m_{v, n}$ is the concentration of vacancy clusters containing $n$ vacancies; $k_{c a s, v n}$ is the rate of production of that size vacancy cluster directly by cascades; $\left(j i, n+1+e_{v, n+1}\right)$ is the sum of the rates with which a cluster of the next larger size acquires interstitials and loses vacancies by evaporation (i.e., the rate at which a cluster of size $n+1$ shrinks to size $n$ ); $j v, n-1$ is the rate with which a cluster of the next smaller size acquires vacancies (interstitial evaporation is neglected); and $d_{c a s, v n}$ is the rate at which a vacancy cluster of size $n$ is dissolved by formation of new cascades. The assumption is made that clusters grow and shrink only via acquisition or loss of single point defects. The first three terms of the right hand side of eq.(2) represent the rate of gain, and the last term the rate of loss, of clusters of size $n$. For interstitial clusters an equation entirely equivalent to eq. (2) is valid. As mentioned above, the set of equations for interstitial and for vacancy clusters of the form of eq. (2) can be integrated using sufficiently small time steps. The coefficients in eq. (2) are recalculated by solving eqs. (1) for steady state defect 
concentrations applicable to the cluster distributions obtained after the previous time step. The sink strengths and fluxes, $\mathrm{ji}_{\mathrm{i}, \mathrm{n}}$ and $\mathrm{j}_{\mathrm{v}, \mathrm{n}}$, are calculated by the effective medium approach as discussed by Brailsford and Bullough [47], which also uses the steady-state approximation with regard to the mobile defects. The thermal emission rate of vacancies from clusters is determined by taking the difference in the energies of clusters of sizes $n+1$ and of $n$ into account. The steady state approximation used for recalculating the coefficients in eq. (2) requires that the lifetinies of free defects are short compared to the lifetimes of immobile clusters.

Although the evolution of the defect cluster distributions have been calculated from the above equations only under a set of rather simple assumptions (e.g., all cascades are of a sir.gle size; defect clusters above minimum sizes are immobile; smaller sizes decompose into single, mobile defects spontaneously; and no dislocation bias) some general trends can be outlined that are expected to hold more generally at low and intermediate temperatures [44]. The cascade parameters used in the calculation are given in table 1.

At low temperatures at which the thermal evaporation of vacancies from small vacancy clusters is unimportant, highly dense cluster distributions evolve within the first few tenths of a dpa. Because more interstitials than vacancies escape from the periphery of the cascades, some interstitial clusters grow to large sizes even in the absence of a dislocation bias. As a corollary, vacancy clusters are prevented from growing to large sizes: no significant swelling can occur. Under these circumstances, interstitials are contributing more than vacancies to thermal mass transport. The development of defect cluster distributions in this temperature regime as illustrated in figure 1 . 
At higher temperatures, thermal evaporation of vacancies from vacancy clusters increases to the extent that vacancy release to the matrix exceeds that of interstitials. This situation corresponds to the "production bias" postulated by Woo and Singh $[48,49]$. As a consequence of the excess vacancy release, some vacancy clusters grow to large sizes and those which have remained three dimensional void embryos may surpass the critical size corresponding to the excess vacancy release to the matrix. This situation corresponds to the onset of void swelling. Interstitial dislocation loops would be prevented from growing to large sizes unless dislocation bias overcomes the excess vacancy flux impinging on interstitial loops. Vacancies contribute more to mass transport than interstitials. An example of the development of the vacancy cluster size distribution in the void swelling regime is shown in figure 2.

The model predicts correctly the features of microstructural evolution at the lower temperatures including the transition to the void swelling regime. However, before the model calculations can be applied to higher temperature irradiations, some of the simplifying assumptions must be removed. In particular, thermal motion of small dislocation loops by glide may be very important because their quasi one-dimensional motion should remove the loops rather efficiently to extended sinks (network dislocations and grain boundaries) as Trinkaus et al. suggest [50].

\section{Outlook}

Although a quantitative description of the evolution of complex microstructures in different irradiation environments may remain an unsolved task for some time, many elements for such a description are now available. The development of defect cluster distributions, dislocation loops and voids can be 
calculated for primary knock-on energy spectra characteristic for given irradiation environments with the model outlined above. The model provides free defect fluxes which are needed for characterizing other microstructural developments such as radiation-induced segregation to stationary sinks, phase separation and precipitate coarsening. In particular, the model could became useful for defining "equivalent" doses for irradiations with different primary knock-on spectra over moderate temperature ranges resulting in "similar" microstructures and, therefore, similar properties. 


\section{References}

[1] Proceedings of the International Conference on Vacancies and interstitials in Metals, C. Abromeit and H. Wollenberger, eds., Materials Science Forum 15-18 (1987).

[2] Proceedings of the Fifth International Conference on Fusion Reactor Materials, R. L. Klueh, R. E. Stoller and D. S. Gelles, eds., North-Holland, Amsterdam (1992).

[3] Proceedings of the International Conference on Physics of Irradiation Effects in Metals, G. Szenes, ed., Materials Science Forum 97-99 (1992).

[4] Proceedings of the Conference on "Evolution of Microstructure in Metals during Irradiation", C. E. Coleman, ed., North-Holland, (1993) in press.

[5] "Physics of Radiation Effects in Crystals", R. A. Johnson and A. N. Orlov, eds., Vol. 13 of the Series ' Modern Problems in Condensed Matter Sciences', NorthHolland, Amsterdam (1986).

[6] M. T. Robinson and I. M. Torrens, Phys. Rev. B9 (1974) 5008.

[7] H. L. Heinisch, J. Nucl. Mater. 117 (1983) 46.

[8] H. L. Heinisch and F. M. Mann, J. Nucl. Mater. 122/123 (1984) 1023.

[9] T. Diaz de la Rubia, R. S. Averback, R. Benedek and I. M. Robertson, Rad. Eff. Def. Sol.113 (1990) 39.

[10] T. Diaz de la Rubia and M.W. Guinan, Phys. Rev. Letters 66 (1991) 2766.

[11] T. Diaz de la Rubia and M. W. Guinan, Mater. Scie. Forum 97-99 (1992), 23.

[12] C.A. English, A. J. E. Foreman, W. J. Phythian, D. J. Bacon and M. L. Jenkins, Mater. Scie. Forum 97-99 (1992) 1.

[13] M. A. Kirk, I. M. Robertson, M. L. Jenkins, C. A. English, T. J. Black and J. S. Vetrano, J. Nucl. Mater. 149 (1987) 21. 
[14] M. Kiritani, Mater. Scie. Forum 15-18 (1987) 1023.

[15] M. Kiritani, Mater. Scie. Forum 97-99 (1992) 141.

[16] M. Wilkins, in: Vacancies and interstitials in metals, A. Seeger, D. Schumacher, W. Schilling and J. Diehl, eds., North-Holland Publishing, Amsterdam, 1970, pp. 485-529.

[17] R. Rauch, J. Peisl, A. Schmalzbauer and G. Wallner, J. Nucl. Mater. 168 (1989) 101 and J. Phys.: Condens. Matter 2 (1990) 9009.

[18] J. Peisl, H. Franz, A. Schmalzbauer and G. Wallner, Mat. Res. Soc. Symp. Proc. Vol. 209 (1991) 271.

[19] K. L. Merkle, in "Radiation Damage in Metals", N. L. Peterson and S. D. Harkness, eds., American Society for Metals, Metals Park, $\mathrm{OH}$, 1976, pp. 58.

[20] K. Urban, M. Wilkins and A. Seeger, in "Radiation-induced Voids in Metals", J. W. Corbett and L. C. Ianniello, eds., U. S. Atomic Energy Commission (1972, pp. 550-562.

[21] F. A. Garner, "High dose effects in simple fcc metals", this proceedings.

[22] J. S. Zinkle, "Microstructural evolution in austenitic stainless steel, transition from low to high dose behavior", this proceedings.

[23] E. A. Little, "Microstructural evolution in ferritic/martensitic steels, transition from low to high dose behavior", this proceedings.

[24] J. H. Evans, in "Patterns, Defects and Materials Instabilities", D. Walgraef and N. M. Ghoniem, eds., Kluver Academic Publishers, 1990, pp.347-370.

[25] Jäger and Trinkaus, J. Nucl. Mater. (1993) in press.

[26] S. Banerjee, K. Urban and M. Wilkins, Acta Met. 32 (1984) 299.

[27] J. Meyer and K. Urban, Acta Met. 33 (1985) 539. 
[28] J. Koike, P. R. Okamoto, L. E. Rehn, and M. Meshii, J. Mater. Res., 5 (1989) 1143.

[29] P. R. Okamoto and M. Meshii, in "Science of Advanced Materials", H. Wiedersich and M. Meshii, eds., ASM International, Metals Park, OH, 1990, pp. 33-98.

[30] G. Martin, R. Cauvin and A. Barbu, in "Phase transformations during irradiation", F. V. Nolfi, Jr., ed., Applied Science Publishers, London and New York, 1983, pp. 47-74.

[31] G. Martin, J. Nucl. Mater. (1993) in press.

[32] H. Wiedersich, in "Physics of Radiation Effects in Crystals", R. A. Johnson and A. N. Orlov, eds., Vol. 13 of the Series ' Modern Problems in Condensed Matter Sciences', North-Holland, Amsterdam (1986), pp. 225-370.

[33] M. J. Norgett, M. T. Robinson and I. M. Torrens, Nucl. Engrg. Des. 33 (1974) 50.

[34] L. E. Rehn, P. R. Okamoto and R. S. Averback, Phys. Rev. B30 (1984) 3073.

[35] L. E. Rehn and P. R. Okamoto, Materials Science Forum 15-18 (1987) 985.

[36] L. E. Rehn and H. Wiedersich, Matfrials Science Forum 97-99 (1992) 43.

[37] A. Müller, M.-P. Macht and V. Naundorf, J. Appl. Phys. 54 (1988) 3445.

[38] V. Naundorf, M.-P. Macht and H. Wollenberger, Materials Science Forum 97 99 (1992) 157.

[39] H. Wiedersich, Rad. Eff. Def. Sol. 113 (1990) 97.

[40] V. Naundorf, J. Nucl. Mater. 182 (1991) 254.

[41] V. Naundorf, M.-P. Macht and H. Wollenberger, J. Nucl. Mater. 186 (1992) 227.

[42] H. Wiedersich, Nucl. Instr. Methods B59/60 (1991) 51.

[43] H. Wiedersich, Mater. Sci. Forum 97-99 (1992) 59. 
[44] H. Wiedersich, J. Nucl. Mater. (1993) in press.

[45] H. Wiedersich, Rad. Effects and Defects in Solids, 12 (1972) 111.

[46] R. Bullough and M. H. Wood, in "Physics of Radiation Effects in Crystals", R. A. Johnson and A. N. Orlov, eds., Vol. 13 of the Series ' Modern Problems in Condensed Matter Sciences', North-Holland, Amsterdam (1986), pp. 189-224.

[47] A. D. Brailsford and R. Bullough, Phil. Trans. Royal Soc. London 302 (1981) 87.

[48] C. H. Woo and B. N. Singh, Phys. Stat. Sol. B159 (1990) 609; Phil. Mag. A 65 (1992) 889.

[49] C. H. Woo, A. A. Semanov and B. N. Singh, this proceedings.

[50] H. Trinkaus, B. N. Singh and A. J. E. Foreman, J. Nucl. Mater. 199 (1992) 1. 
Table 1. Characteristics of reference cascades:

$\begin{array}{ll}\mathrm{T} & =15 \mathrm{keV} \\ \mathrm{N}_{\mathrm{o}} & =200 \\ \mathrm{n}_{\mathrm{io}} & =10 \\ \mathrm{f}_{\mathrm{r}} & =0.77 \\ \mathrm{f}_{\mathrm{vcl}} & =0.22 \\ \mathrm{f}_{\mathrm{icl}} & =0.19 \\ \mathrm{f}_{\mathrm{V}} & =0.01 \\ \mathrm{f}_{\mathrm{i}} & =0.04 \\ \mathrm{f}_{\mathrm{m}} & =15\end{array}$

PKA energy

number of defect pairs (NRT value)

number of interstitials in clusters at formation

fraction of $\mathrm{N}_{0}$ lost by recombination

fraction of vacancies in clusters

fraction of interstitials in clusters

fraction of freely migrating vacancies

fraction of freely migrating interstitials

size of molten zone : $\mathrm{N}_{0} * \mathrm{f}_{\mathrm{m}}=3000$ sites 


\section{Figure captions}

Fig. 1. Development of size distributions of defect clusters at $600 \mathrm{~K}$. The doses are indicated in dpa on the curves. The maxima occur at the size of fornation of the clusters in the cascades. The materials parameters used in the calculations are representative of nickel.

Fig. 2. Development of the vacancy cluster distribution at $660 \mathrm{~K}$. Other parameters as in Fig. 1. Nucleation of voids ceases at higher doses because of a decrease in vacancy cluster size at formation. 


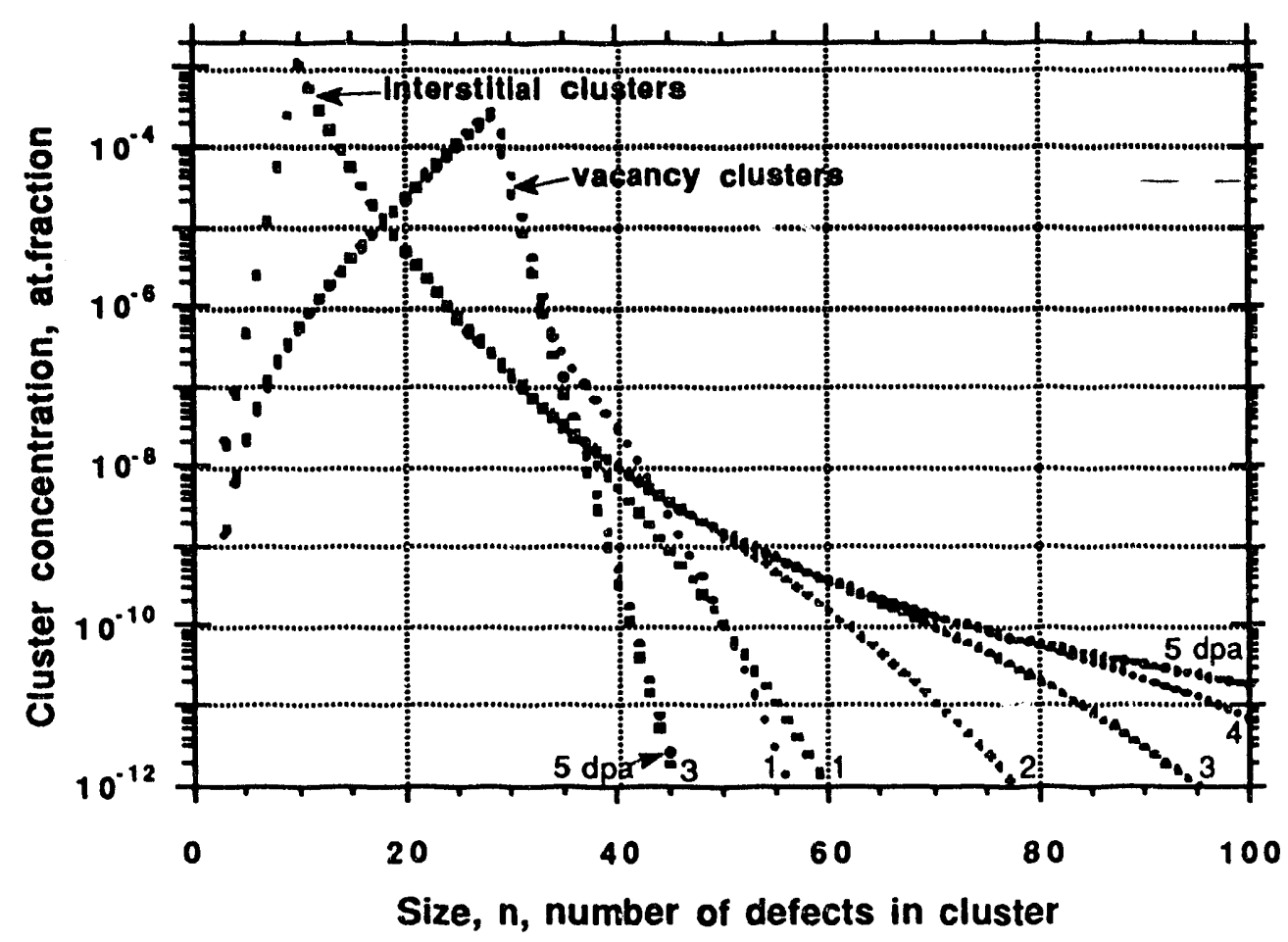

Fig. 1. Development of size distributions of defect clusters at $600 \mathrm{~K}$. The doses are indicated in dpa on the curves. The maxima occur at the size of formation of the clusters in the cascades. The materials parameters used in the calculations are representative of nickel. 


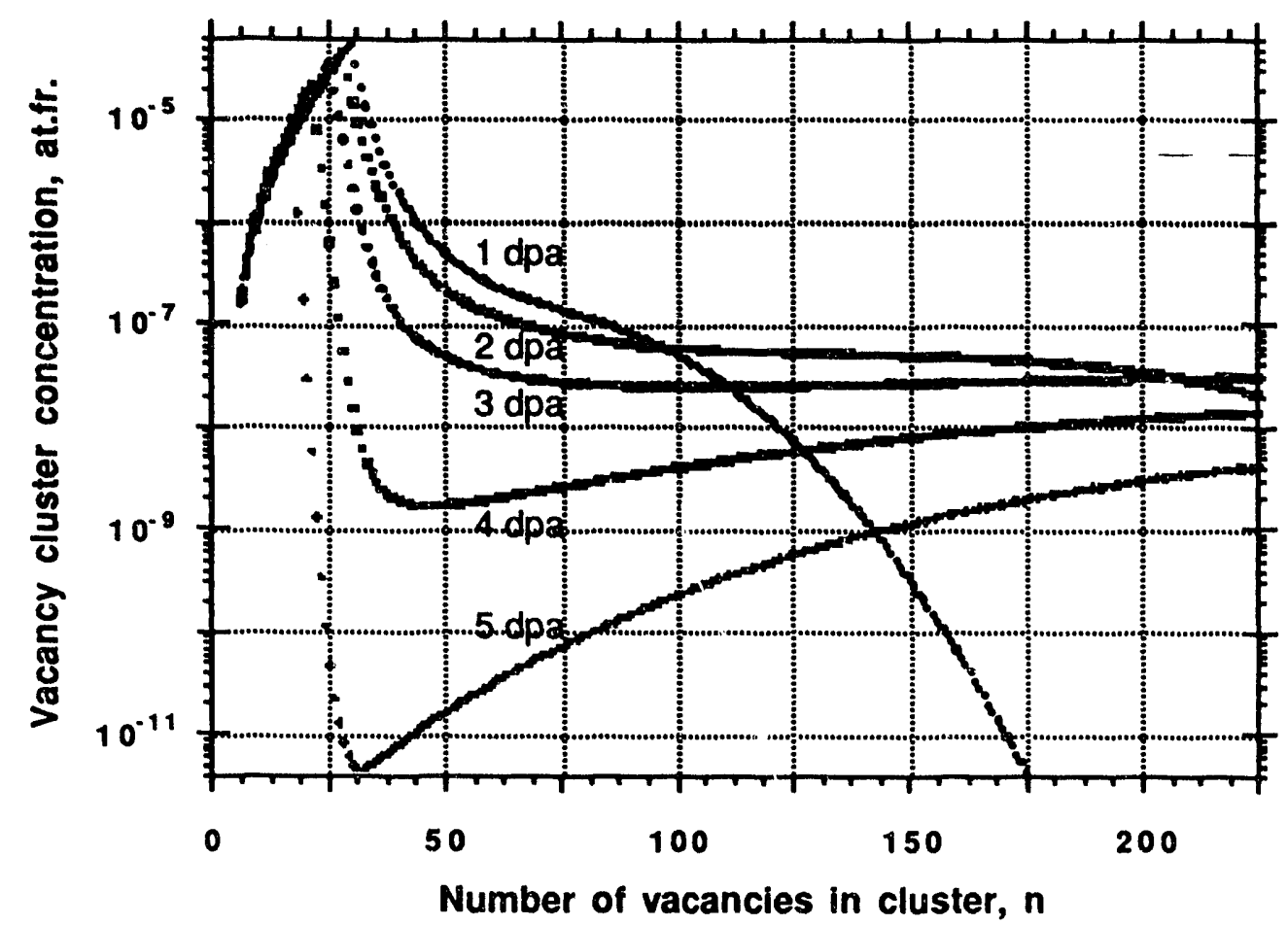

Fig. 2. Development of the vacancy cluster distribution at $660 \mathrm{~K}$. Other parameters as in Fig. 1. Nucleation of voids ceases at higher doses because of a decrease in vacancy cluster size at formation. 

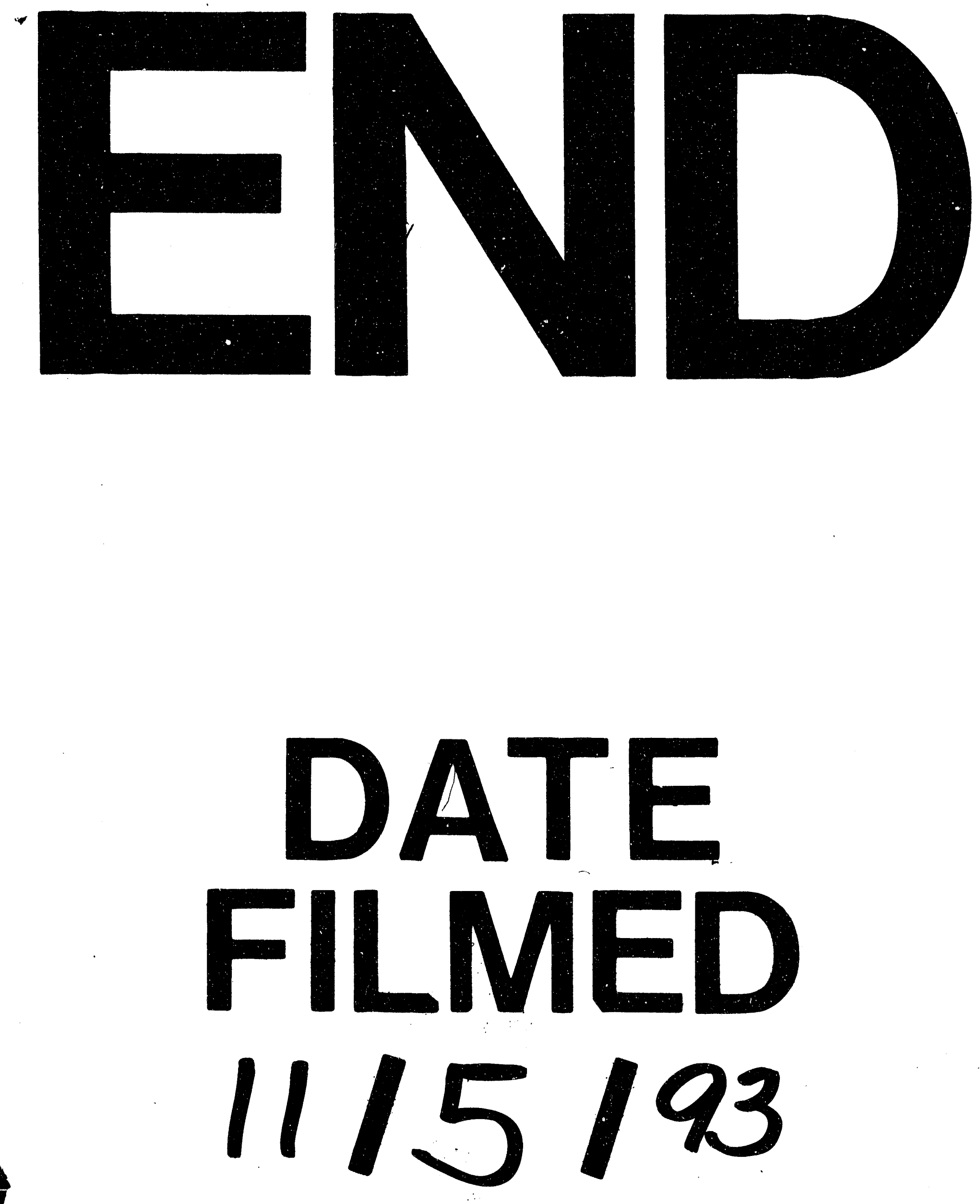

1 